\title{
PARA UNA TEORÍA DE LA HISTORIA DEL DERECHO
}

\author{
FOR A HISTORY OF LAW THEORY
}

\section{CAROLINA UNZUETA OVIEDO*}

\section{RESUMEN}

El artículo hace una reflexión teórica sobre la Historia del Derecho, su sentido, objetivos y fines que la determinan como disciplina autónoma e independiente tanto de la historia como del derecho, considerando el trabajo de autores anteriores. Primero se hace un alcance con respecto a qué se entiende por cada una de estas disciplinas y para luego entrar en la Historia del Derecho propiamente tal. Se extiende la reflexión a algunos ámbitos específicos de análisis, como el tiempo y el espacio, entre otros, para así poder completar el esbozo teórico y científico del tema, planteando un mejor entendimiento del quehacer en cuestión.

Palabras clave: Reflexión, historia, derecho, historia del derecho, tiempo, espacio, fuentes, lenguaje, retórica y magistra vitae.

\section{ABSTRACT}

This article, drawing on the work of other authors, is a theoretical reflection on the History of Law, its sense, objectives and goals that establish it as an autonomous discipline independent of History and Law. Firstly, we address what each one of these features/ aspects means, and then History of Law itself. We expand this reflection to cover some specific points, such as time and space, among others, so as to complete the theoretical and scientific analysis of the subject by offering a better understanding of the task.

Keywords: Reflection, History, Law, History of Law, time, space, sources, language, rhetoric, magistra vitae.

Recibido: 29.12.15. Aceptado: 15.03.16.

* Estudiante del Doctorado en Derecho de la Pontificia Universidad Católica de Chile. Profesora de Historia del Derecho Pontificia Universidad Católica de Chile. Santiago, Chile. Correo electrónico: cunzueta@uc.cl 


\section{INTRODUCCIÓN}

$\mathrm{E}$ L PRESENTE TRABAJO pretende hacer una reflexión sobre la Historia del Derecho, en el entendido de lo necesario que resulta siempre pensar sobre el sentido, objetivos y fines de una determinada disciplina, lo que permite, finalmente, elaborar un mejor entendimiento del quehacer en cuestión. "El renovado estudio de los principios fundamentales de una labor intelectual cualquiera puede servir para encauzarla adecuadamente y definir mejor lo que es y lo que ha de proponerse" (Zorraquín Becú, 1992, p. 461), más aún en el caso de la Historia del Derecho, donde confluyen dos disciplinas claramente diferenciadas, con propósitos y objetivos diversos, pero que en su unión han dado forma a un área del conocimiento autónoma.

Pero el mismo hecho de que se trate de la suma de dos disciplinas lleva a cuestionar aún más la propia existencia de la Historia del Derecho como área autónoma. Se podría pensar que ésta queda en tierra de nadie, en una zona intermedia frente a la cual los autores tienen que entrar a argumentar la propia "razón de ser" de su actividad. Como claramente lo expresa Hans Thieme al decir:

El historiador del derecho es tenido frecuentemente entre los juristas como un buen historiador y entre los historiadores como un buen jurista. No le resulta fácil contentar a unos y a otros. Es un sujeto mixto, con doble ciudadanía, siempre entre dos Facultades, al margen de su propia casa, arrastrando en dos zonas la carga, que hoy percibimos mejor que nunca, del caminante fronterizo. Incómodo a los historiadores por su mentalidad jurídica. Motivo de escándalo para los estudiosos del derecho por su vocación a retrotraer históricamente el estado de las cuestiones. A menudo no sabe cuál es en realidad su patria ni de dónde es ciudadano (Thieme, en Escudero, 1995, p. 33).

Con el tiempo se ha ido estructurando y delimitando esta disciplina que se elabora a dos bandas, y que depende mucho de la inclinación o cercanía de quien la practique, con qué área se vinculará más o menos, si con la Historia o con el Derecho. Por ello es interesante ver qué han dicho los autores con respecto a ella y su desarrollo, de modo que dejaremos que sean ellos mismos quienes nos guíen a través de estas reflexiones y nos muestren sus cuestionamientos y respuestas sobre el sentido de la Historia del Derecho, como bien nos introduce en el tema Paolo Grossi:

Teniendo una conciencia lúcida de todo esto, nosotros nos colocaremos ante un objeto y una finalidad muy determinados: seguir, entre lo 
medieval, lo moderno y lo postmoderno, una dimensión de la historia generalmente descuidada, la dimensión jurídica; una dimensión que, aunque sumida en la globalidad del devenir histórico, cuenta con su propia autonomía, que a veces mantiene fuertes vínculos con el poder político y que a él se somete, pero que sobre todo en las manifestaciones de la praxis de cada día y de la reflexión científica, tiene también la fuerza y la capacidad de recorrer caminos propios (Grossi, 2008, p. 14).

Teniendo esta problemática en mente se hace más patente la necesidad del presente ejercicio que nos permita delinear la historia jurídica, clarificar su campo de acción y comprender mejor su sentido y finalidad. Todo en el entendido de que hablamos de una disciplina con un doble carácter y que por lo mismo debemos, primero, hacer un alcance sobre la Historia y el Derecho por separado, para luego entrar de lleno al producto de su unión, la Historia del Derecho.

\section{LA HISTORIA}

Para intentar definir la Historia se suele hablar de ella como de los hechos del pasado del hombre, lo que sería la realidad pretérita; pero también se entiende por Historia el estudio y el conocimiento científico de dicha realidad. Y el escribir esos acontecimientos es historiografía.

De la meditación de los autores sobre su labor surge una rica discusión sobre el sentido de la Historia, y son ellos entonces los que han definido su propio quehacer. Para Johan Huizinga (1994, p. 98), la "Historia es la forma espiritual en que una cultura se rinde cuentas de su pasado", es decir, busca comprender y dar sentido a la vida del hombre. El mismo autor señala que la definición debe ser precisa para apuntar a la esencia misma de la disciplina, pero su misma brevedad plantea más interrogantes con respecto a ella. En un sentido un poco opuesto encontramos la definición de Lucien Fevbre, ya que él prefiere dar una explicación más acabada para no dar cabida a malas interpretaciones. En este sentido plantea que "la historia es el estudio científicamente elaborado de las diversas actividades y de las diversas creaciones de los hombres de otros tiempos, captadas en su fecha, en el marco de sociedades extremadamente variadas $y$, sin embargo, comparables unas a otras (el postulado es de la sociología); actividades y creaciones con las que cubrieron la superficie de la tierra y la sucesión de las edades" (Febvre, 1992, p. 41).

Aquí tenemos un par de aspectos claves a considerar. Por ejemplo, que la Historia no se califica como ciencia, sino como un estudio científicamente 
elaborado. La ciencia sirve como modelo para los historiadores, aportando su método, rigurosidad y algunos principios; hay incluso una coincidencia en el método científico con el histórico, al menos de los que no se pueden reproducir en el laboratorio (Gaddis, 2004, p. 66). Pero más que una ciencia la Historia es una disciplina más cercana al Arte, por su esencia y finalidad.

Otro punto fundamental es el hombre, el que se considera como objeto único de la Historia, comprendido "en el marco de las sociedades que son miembros" (Febvre, 1992, p. 41). Hay aspectos del pasado donde el hombre no tuvo cabida y que pueden ser muy interesantes, pero que su estudio corresponde a otras disciplinas, generalmente científicas, y no hay que olvidar que la historia "en cualquiera de sus ramas y modalidades, se refiere siempre al hombre y que al ser humano no se le puede llegar a comprender si no se ponen en juego todas las dimensiones que lo constituyen" (Fontana, 1992, p. 40).

El hombre inserto en una sociedad del pasado que llega hasta nosotros gracias a que en el presente nos preguntamos por él y queremos desentrañar su momento. Pero el cómo llegamos a él presenta otra clave con respecto a la construcción de una definición de Historia. En ese sentido Edward Carr la presenta como "un proceso continuo de interacción entre el historiador y sus hechos, un diálogo sin fin entre el presente y el pasado" (Carr, 2001, p. 76). Se trata de un diálogo, entonces, donde se plantea un problema y luego se formula una hipótesis que permite guiar la investigación para que lleve a resolver la problemática planteada. Pero es más que un diálogo, como acota Fernand Braudel (2009, p. 9) al decir que "la historia no es otra cosa que una constante interrogación a los tiempos pasados en nombre de los problemas y curiosidades -e incluso las inquietudes y las angustias- del presente que nos rodea y nos asedia", de manera que el hombre no sólo quiere responder a alguna problemática planteada, sino que va incluso más allá buscando sentido a su presente en su cuestionamiento del pasado.

Vemos cómo el historiador se mueve así del presente al pasado y viceversa, y ¿para qué? Para comprender ese pasado y, especialmente, el presente, pero más aún, para iluminar el futuro. El historiador tiene una visión más amplia sobre los acontecimientos, lo que lo ayuda a entenderlos y presentarlos de manera más clara y útil; lo que hace que, finalmente, la Historia resulte el mejor método para aprender del y el pasado.

Lo que nos lleva a un último aspecto a considerar: la Historia como maestra de vida. Ya los clásicos la entendían así y supieron captar su utilidad e importancia como medio de enseñanza -política y moral, básicamente-. En este sentido Polibio señala que "para los hombres no existe 
enseñanza más clara que el conocimiento de los hechos pretéritos" (Polibio, 1981, 1.1). Y a pesar de que hoy no se considera a la historia como maestra en el mismo sentido que los antiguos, igual se reconoce su utilidad pedagógica: "Estamos destinados a aprender del pasado, hagamos o no el esfuerzo pertinente, pues es la única base de datos que tenemos" (Gaddis, 2004, p. 26), es decir, se busque o no, la Historia "es progreso a través de la transmisión, de una generación a otra, de las habilidades adquiridas" (Carr en Gaddis, 2004, p. 26).

\section{DERECHO}

Así como definir la Historia presenta sus complejidades, lo mismo ocurre con el Derecho, debido a la multiplicidad de factores históricos, culturales y filosóficos que convergen en su contenido. Los mismos romanos evolucionaron desde el concepto de ius, entendido como el arte de lo bueno y equitativo, al de directum, que hace de la ley el camino a seguir (de donde proviene la voz Derecho, Direito, Dret, Droit, Diritto) (Coronas González, 1996, p. 11). La palabra derecho significa, entonces, lo que es recto, no torcido; y ya en el mundo romano correspondía a los jueces determinar lo que es recto o no, con efectos sociales.

¿Qué debemos entender entonces por Derecho? Una buena definición nos la da Alfonso García Gallo cuando señala que "en líneas generales puede decirse que es un conjunto de principios y normas que regulan la vida social, manteniendo el orden en ella" (García Gallo, 1950, p. 6). Toda sociedad, y de cualquier época, se ordena y dirige por normas, entre las que se distinguen las morales o de sentido religioso, las propiamente jurídicas y las normas o usos sociales. La distinción entre ellas siempre ha causado problemas, ya que los límites a veces son difusos. Hay normas morales cuya contravención, el pecado, coincide con el delito o ruptura del orden jurídico (ej.: el homicidio), pero hay otras que no tienen que ver con el derecho; al contrario, hay normas jurídicas que no tienen valor moral (ej.: leyes del tránsito). Lo que no quiere decir que moral y derecho se encuentren en campos opuestos ya que el derecho "suele acomodarse a los principios morales, más o menos elásticos, que imperan en la sociedad" (Escudero, 1995, p. 21). Entre las normas morales y los usos sociales puede haber más o menos coincidencia, dependiendo del tipo de sociedad; mientras más religiosa, más relación y confusión. Por último, el límite entre derecho y usos

sociales nunca ha sido muy claro, "porque junto al derecho escrito existe un 
derecho consuetudinario, más fácilmente confundible con determinados usos (y) porque el mismo derecho escrito ha incidido en esos usos" (Escudero, 1995, p. 21).

Frente a esta dificultad de diferenciación, cómo, entonces se pueden identificar las normas jurídicas. Aquí entra en escena la coactividad de la norma, a pesar de que no es un punto absoluto. "Con ciertas cautelas cabría admitir que las normas jurídicas, a diferencia de las morales o de los usos, son aquellas cuya vulneración es perseguida por el grupo social organizado mediante procedimientos coactivos que se traducen en pena" (Escudero, 1995, p. 21). De manera que mientras más desarrollada la sociedad, más claridad habrá en la diferenciación y delimitación de las normas según el tipo de que se trate.

Pero el Derecho es más que un conjunto de normas. Sin duda su manifestación más llamativa son los actos legislativos, los cuales implican poder y ordenación, pero aquí hay un reconocimiento más amplio ya que "es la misma sociedad la que se auto-organiza percibiendo ciertos valores históricos, trazando sobre ellos algunas reglas y observándolas en la vida de cada día" (Grossi, 2008, p. 15). De modo que las sociedades son entes claves para entender el Derecho, ya que éste "pertenece a la sociedad y, por lo tanto, a la vida, expresa a la sociedad más que al estado, es el tejido invisible que vuelve ordenada a nuestra experiencia cotidiana, consintiendo la convivencia pacífica de las recíprocas libertades. Es identificable, pues, con un auténtico auto-salvamento de la sociedad" (Grossi, 2008, p. 15).

Resulta incuestionable que el derecho está inscrito en el hombre y es una dimensión más de la vida de él, por lo que estaría antes de que aparecieran las normas jurídicas propiamente tales. Pero, a la vez, el Derecho se concretiza en un ordenamiento positivo que regula y ordena dicha sociedad; y es esa forma concreta la que constituye la dogmática jurídica propiamente tal.

Dando un paso más en lo que es el Derecho podemos agregar entonces que éste se compone por leyes (normas en términos generales) y hechos jurídicos, los que al ser interpretados por jueces, profesores y juristas, producen jurisprudencia y doctrina; con lo cual tenemos completo el cuadro, dando claridad al conjunto. Sólo entonces se puede entender lo jurídico, como referido a todo lo que atañe al derecho.

\section{HISTORIA DEL DERECHO}

Partiendo de la base de que el Derecho, como todo lo relacionado con el hombre, está afectado por el paso del tiempo, su evolución puede ser objeto 
de estudio. Y es aquí donde nos encontramos con la Historia del Derecho, la que "se detiene a analizar el pasado jurídico, entendiendo por tal, no el estudio estático y aislado de algún código antiguo o de una determinada institución ya muerta, materias que caen dentro de la arqueología jurídica, sino el estudio dinámico de la idea del derecho y de sus realizaciones" (Eyzaguirre, 1962, p. 17). Es decir, estudiaremos el Derecho en su desarrollo histórico y no simplemente como un régimen jurídico del pasado.

Llegar a esta conclusión tomó tiempo ya que no siempre se ha tenido conciencia de la historicidad del Derecho. Será gracias a la Escuela Histórica alemana de Savigny que el Derecho será "concebido como una creación histórica del hombre, una emanación de su fuerza creativa, exponente, al igual que otras manifestaciones de su cultura o lengua, del espíritu popular (volkgeist)" (Coronas González, 1996, p. 14). Lo que permite que el Derecho comience a ser estudiado como proceso histórico que se realiza en una sociedad y época concreta. En un principio la Escuela Histórica del Derecho Alemana fue una escuela de juristas, pero desde mediados del siglo XIX sus miembros se fueron acercando a la Historia "y paulatinamente la Historia del Derecho tiende a perder su configuración de ciencia jurídica para pasar a ser considerada una rama de la Historia general" (Montanos Ferrín y Sánchez-Arcil, 1991, p. 7).

Se entiende entonces el desarrollo del Derecho como parte de la Historia de la humanidad, y como tal forma parte de la experiencia y de las necesidades sentidas en cada época. En ese sentido son clarificadoras las palabras de un historiador del Derecho inglés de fines del siglo XIX, Oliver Wendell Holmes, cuando afirma que:

En el derecho cristaliza la historia del desarrollo de una nación a través de los siglos y por ello aquel no puede contemplarse como si contuviese únicamente los axiomas y corolarios de un libro de matemáticas. Para conocer el derecho debemos saber lo que ha sido y lo que tiende a ser. Es necesario consultar alternativamente la historia y las teorías legales vigentes. Pero lo más difícil consiste en comprender la conjunción de ambas en los productos nuevos de cada etapa. La sustancia de la ley en cualquier época casi siempre corresponde, al menos hasta ahora, a lo que en ese momento se entiende como conveniente; sin embargo, sus formas y mecanismos y el grado en el que pueden lograr los resultados deseados dependen en gran medida de su pasado (Holmes, en Aguilera, 1996, p. 19).

Con esta comprensión de la Historia del Derecho como un ámbito clave en el desarrollo de las sociedades se entiende entonces su función, que ha 
sido concebida de "alta cultura", en el sentido de que en la formación de las naciones y sus instituciones no pueden prescindir de sus antecedentes histórico-jurídicos. Como bien señala el historiador del derecho italiano Paolo Grossi:

No olvidaremos nunca que el derecho no pertenece sólo a la superficie de la sociedad, sino que es, al contrario [...], una realidad radical, es decir, conectada a las raíces profundas de aquella. No olvidaremos nunca que, antes que una orden, el derecho es una mentalidad, es decir expresa una costumbre y la ordena, expresa los valores de una civilización y -al ordenarla- la salva (Grossi, 2008, p. 16).

El Derecho ordena y salva a una sociedad, y esto no sólo debido a que las normas jurídicas “ordenan”, sino porque, además, el Derecho es una estructura que sostiene todo el entramado social. Justamente el profesor de Historia del Derecho Bernardino Bravo afirmó que su interés por la disciplina surgió cuando:

Leí la afirmación de un historiador alemán, Heinrich Metteiz, quien decía que la historia del derecho es la puerta adecuada para entrar a las demás ramas de la historia, porque cuando un historiador empieza a trabajar se encuentra con un caos de datos que hay que ordenar de alguna manera, y esa ordenación muchas veces es bastante arbitraria; en cambio -en materia jurídica-, el derecho es en sí mismo una estructura, y no hay más que reconstruir la estructura y no construir una. Eso me fascinó (en Rodríguez Medina, 2010, p. E5).

Se conjugan así varios elementos importantes para entender la Historia del Derecho como una disciplina autónoma tanto de la Historia como del Derecho; cuyo objeto de estudio es la normativa destinada a resolver problemas concretos de convivencia y todo lo que ello implica. De tal manera que "el arte del historiador del Derecho, como el de todo otro historiador, está en saber combinar los datos, incluso mínimos, con el amplio trazado de tradiciones y coyunturas: una microscopia de los textos con la macroscopia diacrónica y sincrónica de las instituciones" (D’Ors, 1999, p. 33).

El historiador del Derecho tiene que saber plantear un problema y luego saber elegir y combinar los datos e información obtenida para poder elaborar respuestas satisfactorias en el ámbito histórico-jurídico, pero que se inserten y tengan coherencia en un ámbito mayor, ya que el hombre no vive en una sociedad dividida en compartimentos estancos, sino todo lo contrario, "los hombres toman conciencia de los problemas de la sociedad, y 
los resuelven, en el terreno de 'las normas jurídicas, políticas, religiosas, artísticas o filosóficas"' (Fontana, 1992, p. 102). En este contexto se entiende entonces el planteamiento del jurista italiano Emilio Betti cuando plantea una relación "entre Historia y Derecho como plenitud práctica de la relación entre cultura y sociedad (lo histórico es aquello que lleva la historia consigo, reconociéndola por medio de la Filología y haciendo uso de ella como un instrumento problemático). En cuanto al Derecho, es solución de problemas de convivencia. La solución es vinculante sobre el plano práctico (para el sistema, para el poder), pero son los problemas los que han de llevar al jurista a reconocerlos en su historicidad. Por ello viene asignada al jurista (y a la doctrina), en todas las épocas, una función política, una tarea, una misión social, en definitiva, una actividad de crítica de la sociedad" (Betti, 2009, p. XXV).

Más allá de estas reflexiones que nos permiten entender el sentido de la Historia del Derecho, podemos plantear en concreto su objetivo, que sirve para definir su quehacer, el que sería "el estudio del Derecho del pasado, de su evolución y de su devenir histórico que incluye el estudio de la historia de la legislación y del pensamiento jurídico, el análisis del derecho consuetudinario y de la labor jurisprudencial, [...] (dejando) a un lado, tanto el estudio del ordenamiento jurídico vigente como las parcelas propias de la Filosofía del Derecho" (Gómez Rojo, 2003, p. 87). Y su producto será una Historia del Derecho centrada "en destacar la correspondencia del Derecho con la política, la cultura, la geografía, la economía o la realidad social de otras épocas, (haciendo) patente el carácter mutable del Derecho y (otorgando) al investigador y al estudiante una visión de la realidad jurídica más completa y crítica desde una perspectiva histórica" (Gómez Rojo, 2003, p. 227).

Teniendo así claros el sentido y objetivos de la Historia del Derecho resulta interesante hacer algunos alcances en ciertos conceptos que se vinculan tanto con la Historia como con el Derecho y que permiten profundizar en el ejercicio teórico del tema en cuestión.

\section{HISTORIA DEL DERECHO Y TIEMPO}

Como ya lo señaló Heródoto, la Historia se escribe "para evitar que, con el tiempo, los hechos humanos queden en el olvido" $(1984,1.1)$. Se quiere rescatar los hechos de determinado momento, de manera que el factor tiempo es clave para la comprensión de los fenómenos históricos y también para la explicación metodológica a seguir. Es decir, tenemos que tener clara la 
cronología en que se insertan los hechos, pero además es útil construir periodificaciones que ayuden a la comprensión del tema estudiado, teniendo claro lo cuestionables que puedan ser estas divisiones temporales, pero a la vez, lo prácticas que resultan. Además, dentro de una periodificación caben tanto los hechos más remotos, como también la historia del tiempo presente.

Justamente la Historia del Derecho, al ser una disciplina histórica, ha utilizado el Método Histórico, según el cual la materia se divide en grandes períodos históricos y dentro de cada período se estudia el conjunto de las instituciones jurídicas. Este método permite apreciar mejor la evolución de los sistemas jurídicos, ayudando así a su cabal comprensión. Pero por un asunto de utilidad práctica y pedagógica la Historia del Derecho no se amolda a los períodos de la Historia general, sino que se divide conforme a las etapas de su propio desarrollo, teniendo claro que los límites entre un período y otro nunca son rígidos (García Gallo, 1950, p. 8). Además, dependerá de la Historia del Derecho de cada país los períodos a tratar y, por lo tanto, su delimitación e interconexiones con las historias de otros países.

\section{HISTORIA DEL DERECHO Y ESPACIO}

La Historia del Derecho no sólo se inserta en el tiempo, sino que también en un espacio determinado, así vemos que en la periodificación temporal se incluye también el aspecto geográfico. Y la "determinación del ámbito espacial de la disciplina tiene singular importancia a la hora de llevar a cabo un análisis científico de la misma" (Gómez Rojo, 2003, p. 7), ya que permitirá reconstruir el Derecho y las instituciones históricas de cada nación. Por otra parte, el Derecho, "creado para ordenar la vida social del hombre, tiene un ámbito espacial correspondiente al grado de desarrollo histórico de la sociedad. A los círculos familiares o tribales de los estadios primitivos se han ido superponiendo los territorios de base nacional y aun supranacional de los tiempos posteriores, ofreciendo una visión compleja de la ordenación jurídica articulada en diferentes estratos o niveles" (Coronas González, 1996, p. 17).

\section{HISTORIA DEL DERECHO Y FUENTES}

Podemos considerar como fuentes de la Historia del Derecho "todo fenómeno apreciable físicamente, que puede dar noticias de la esencia, suerte 
o contenido de un principio jurídico" (Eyzaguirre, 1962, p. 24). A lo que tenemos que sumar el uso de la Heurística, es decir, la ciencia que tiene por objeto el adecuado manejo, ordenamiento y depuración crítica de las fuentes de conocimiento histórico jurídico (Eyzaguirre, 1962, p. 17; Coronas González, 1996, p. 19). Dentro de las fuentes se distinguen las directas, inmediatas o jurídicas, propiamente tales, como la ley, la costumbre, la jurisprudencia y la doctrina, por nombrar a las más importantes, pero incluyendo a todas aquellas que informan directamente sobre el Derecho. Y las fuentes indirectas, mediatas o no jurídicas, donde caben las fuentes de la Historia general y, en ese sentido, todo lo que nos dé un indicio histórico según lo que estemos investigando, y donde, además, resultan fundamentales las ciencias auxiliares como la numismática, la epigrafía, y un largo etc., y el uso de otros métodos como el comparativo o el estadístico (Gómez Rojo, 2003, pp. 129-134).

En la medida en que se abarca un aspecto de la Historia jurídica se comienza a vislumbrar la complejidad del mundo en que se inserta y, por lo mismo, se aprende a valorar las fuentes que nos entregan las más variadas informaciones en relación al tema. Y mientras más amplia sea la mirada a las fuentes, más ricas y complejas serán las respuestas que se obtengan. Por ello, entonces, resulta útil y beneficioso seguir "a legisladores, jueces, científicos, comunes hombres de negocios en una historia marcada por una dialéctica perenne entre localismo/particularismo y universalismo, donde el derecho revela su carácter de realidad que asoma en la superficie de la cotidianidad desde las raíces profundas de una civilización, y que por lo tanto sabe expresarla con su cifra más auténtica, y que revela asimismo -en virtud de esta capacidad- su posible autonomía de las decisiones contingentes del poder político" (Grossi, 2008, p. 14).

\section{HISTORIA DEL DERECHO Y LENGUAJE}

"Lo mismo que hemos puesto la libertad de expresión y la verdad como objetivos del historiador, así también debe ser uno el principal propósito de su lenguaje: explicar el tema con claridad y mostrarlo con la mayor evidencia posible, sin emplear palabras oscuras y fuera de uso, ni tampoco términos vulgares y propios de tenderos, sino que pueda entenderlas la mayoría y elogiarlas las personas cultas" (Luciano, 1990, p. 44). Lo dijo Luciano ya en la antigua Roma refiriéndose al lenguaje que debían usar los historiadores, pero cabe perfectamente hoy en día para quien escriba cualquier Historia. 
Y la advertencia deben tenerla en cuenta como un especial desafío los historiadores del Derecho debido a que de por sí el lenguaje jurídico es complejo, "ya Goethe (en Poesía y verdad) reprochó al lenguaje jurídico que por sus ataduras formalistas careciera de auténtica libertad" (Escudero, 1995, p. 23). Con el Derecho se parte de la base que estamos frente a un lenguaje sumamente técnico, de manera que para poder entenderlo se necesita de un conocimiento básico del mismo. Pero una cosa es el lenguaje técnico específico y otra complejizarlo aún más casi por gusto profesional. La crítica la encontramos entre los propios hombres de Derecho, como cuando Grossi reclama que "no ha sido fácil asegurar la claridad (de su obra) ante un vocabulario y un ideario -como los de los juristas- muy herméticos en su especialidad" (Grossi, 2008, p. 11).

A la dificultad técnica del lenguaje se suma su especificidad en cada época ya que cada sociedad tiene sus particularismos, y mientras más elaborado y desarrollado su derecho, más complejo será su lenguaje. Pero, además, este lenguaje irá variando y evolucionando con el tiempo, de modo que será necesario analizar también el devenir de los conceptos jurídicos para poder entender su implementación en la práctica.

De este punto surge otra problemática respecto del lenguaje en el ámbito del Derecho. Y es que la lengua le sirve de aparato de expresión, pero también tiene que garantizar su comprensión en el tiempo. El ritmo jurídico es pausado, ya que el Derecho evoluciona más lentamente que los cambios ideológicos, económicos y sociales, porque justamente lo que se le pide es que garantice estabilidad. En ese sentido se destaca el paralelismo entre la evolución del Derecho y la del idioma, "mientras en lo genéricamente histórico acontece la individualidad e irrepetición de los hechos, la seguridad y la mutua comprensión exigen del Derecho y del idioma una mayor fijeza y un cambio más lento en su proceso evolutivo" (Escudero, 1995, p. 23).

\section{HISTORIA DEL DERECHO Y RETÓRICA}

"Pero volviendo a lo mío, ¿̇os dais cuenta hasta qué punto escribir historia es competencia del orador? Y casi diría que lo es en grado superlativo, si se atiende al fluir del discurso y la variedad. Mas en parte alguna la veo tratada fuera en las reglas que dan los rétores, pues esas reglas están a la vista de todos. ¿Pues quién ignora que la primera ley de la historia es no atreverse a mentir en nada? ¿Y a continuación el atreverse a decir toda la verdad? ¿Y al escribirla no haya sospecha de simpatía o animadversión?" (Cicerón, 2002, 
2.62). Es Cicerón, un hombre vinculado al mundo jurídico, quien está hablando sobre cómo escribir Historia, estableciendo pautas que calzan perfecto para la Historia del Derecho, baste recordar la estrecha relación entre Derecho y retórica, desde los romanos en adelante.

Ya los antiguos le pedían a su Historiografía estar bien escrita y cumplir con ciertas pautas y rangos, para lo cual era fundamental entonces el buen manejo de la retórica. Exigencia que se le puede hacer también a la Historia del Derecho, considerando que los elementos de la retórica resultan de suma utilidad para poder elaborar una historiografía jurídica de calidad. La retórica incluye: estilo, invención, orden, memoria y dicción, todos elementos que al ser usados apropiadamente permiten dar sentido a la obra. Esto porque al tener los materiales reunidos, corresponde seleccionarlos y ordenarlos de tal manera que permitan reconstruir, organizar y explicar el problema estudiado. Incluso la invención tiene cabida en la Historia del Derecho, ya que no se trata de establecer conclusiones de la nada, sino de permitir al historiador usar la imaginación con las fuentes que maneja para reconstruir de la mejor manera posible ese pasado que está tratando de explicar. Y todo con un estilo claro, profundo y elegante.

\section{HISTORIA DEL DERECHO MAGISTRA VITAE}

"En efecto, ¿hay algo tan importante como el derecho de la ciudad?" (Cicerón, 1989, 4.14). Nuevamente Cicerón nos introduce en el tema de la importancia y trascendencia de la Historia de Derecho como disciplina humanista, al darle una valoración fundamental al Derecho en la vida del hombre. Más aún, esta idea Cicerón la profundiza al señalar que "las leyes sirven para mantener la res pública, pero es ésta la que, con su perfección, proporciona al hombre una vida feliz" (Cicerón, 1984, 4.3).

Se podría contraargumentar que la recomendación viene de cerca, tratándose de un abogado romano quien lo dice. Pero la valoración positiva y la necesariedad del Derecho en la vida del hombre la encontramos reiteradas veces, lo que permite aquilatar entonces la propuesta.

$\mathrm{Al}$ respecto, es destacable el alcance que hace el historiador inglés Edward Gibbon al introducir su capítulo sobre el Derecho Romano en su magna obra Decadencia y caída del Imperio Romano (1776-1789): "me detendré a contemplar los principios de una ciencia tan importante para la paz y la felicidad de la sociedad. Las leyes de una nación constituyen la parte más instructiva de su Historia" (Gibbon, 2006, p. 63). 
Aquí encontramos quizá la función más importante de la Historia del Derecho, la formación. Sin duda destaca "el elemento cultural que aporta a la formación teórica de los futuros juristas" (Gómez Rojo, 2003, p. 228), y no sólo a ellos sino que a la sociedad toda, ya que al ser el derecho un ámbito $\tan$ fundamental de la sociedad aporta también en gran medida a su formación y desarrollo cultural. Como bien acota Grossi: "Desde siempre he tenido la conciencia de que la mirada del historiador del Derecho debía ser más amplia, tanto para alcanzar las épocas medieval, moderna y contemporánea, como para abarcar todo el espacio europeo (como mínimo el europeo). Sólo de esta manera la Historia del Derecho podía cumplir (tal como la cumple hoy) su inexcusable función formativa" (Grossi, 2008, p. 12).

La Historia del Derecho en particular, y la Historia en términos generales, educan, cumpliendo así con la clásica idea de la Historia acuñada por Cicerón, que la concibe como "testigo de los tiempos, luz de la verdad, vida de la memoria, maestra de la vida, mensajera de la antigüedad".

Se reconoce la función de la Historia como maestra de vida, pero en el sentido de que conocemos el pasado para conocer mejor nuestro presente, "ya que en el autoconocimiento se encuentra el fundamento de nuestro vivir futuro" (Iglesias Ferreirós, 1996, p. 15).

\section{CONCLUSIONES}

A modo de conclusión baste volver a la idea inicial del trabajo, donde se planteó como objetivo hacer una reflexión sobre la Historia del Derecho concebida como una disciplina humanista que se configura sobre la base de la unión de la Historia con el Derecho, y lo necesario que resulta pensar sobre el oficio al que uno se dedica y averiguar también lo que otros han meditado al respecto. En este sentido es importante conocer el trabajo intelectual de los congéneres para poder entrar en el círculo de pensamiento y discusión de la propia disciplina y, a la vez, poder recoger esa sabiduría que otros con anterioridad y clarividencia ya han adelantado al respecto.

Y así como en la justificación de una obra biográfica se valora el aporte de la vida de un artista, poeta o escritor al señalar que: "Pues éste me parece el cometido principal de la biografía: representar al hombre en las circunstancias de su época y mostrar en qué medida se resiste a ellas, en qué medida le favorecen, cómo a partir de ellas se ha formado una visión del mundo y de los hombres y cómo, si se trata de un artista, poeta o escritor, ha proyectado esta visión al exterior" (Sala, en Goethe, 1999, p. 21). 
También podemos hacer totalmente válida esta apreciación para valorar el aporte que puede hacer la obra de un Historiador del Derecho para sus pares y para la sociedad en términos generales.

Por otra parte, resulta interesante rescatar el valor que se puede dar a la Historia del Derecho desde la perspectiva vocacional. Pensar una disciplina lleva a buscar justificativos de la propia profesión y, en última instancia, de la propia vocación que determina nuestras actividades cotidianas. La auténtica vocación nos lleva a practicar un oficio con rigor y honradez ya que "para trazar planos, vastos planos, amplios planos, hacen falta espíritus vastos y amplios. Se precisa una visión clara de las cosas. Es necesario trabajar de acuerdo con todo el movimiento de su tiempo. Hay que tener horror de lo pequeño, de lo mezquino, de lo pobre, de lo atrasado. En una palabra: hay que saber pensar" (Febvre, 1992, p. 58).

Por último, cumplir con la propia vocación nos ayuda en la realización personal, lo que sencillamente nos hace más felices. Y como bien dice Lucien Febvre:

Me gusta la historia. No sería historiador si no me gustara. Cuando el oficio que se ha elegido es un oficio intelectivo resulta abominable dividir la vida en dos partes, una dedicada al oficio que se desempeña sin amor y la otra reservada a la satisfacción de necesidades profundas. Me gusta la historia y por eso estoy contento al hablaros hoy de lo que me gusta (Febvre, 1992, p. 37).

\section{REFERENCIAS}

Aguilera Barchet, B. (1996). Introducción jurídica de la Historia del Derecho. Madrid: Civitas.

Betti, E. (2009). La interpretación jurídica. Santiago: Legal Publishing.

Braudel, F. (2009). El Mediterráneo. El espacio y la historia. México, FCE (1ª ed. francés, 1985).

Carr, E. (2001). ¿Qué es la historia? España: Ariel (1a edición en inglés, 1961).

Cicerón, M. T. (1984). Sobre la República. Madrid: Gredos.

Ciceron, M. T. (1989). Las Leyes. Madrid: Alianza.

Ciceron, M.T. (2002). Sobre el Orador. Madrid: Gredos.

Coronas González, S. M. (1996). Manual de historia del derecho español. Valencia: Tirant lo Blanch.

D’Ors, Á. (1999). Nueva Introducción al estudio del Derecho. Madrid: Civitas.

Escudero, J. A. (1995). Curso de historia del derecho. Madrid: Solana e Hijos.

Eyzaguirre, J. (1962). Historia del derecho. Santiago: Editorial Universitaria. 
Febvre, L. (1992). Combates por la historia. Barcelona: Ariel (1 ${ }^{\text {a }}$ ed. francés, 1953).

Fontana, J. (1992). La historia después del fin de la historia. Barcelona: Crítica.

Gaddis, J. L. (2004). El paisaje de la historia. Como los historiadores representan el pasado. Barcelona: Anagrama (1 ${ }^{\text {a }}$ ed. inglés, 2002).

García Gallo, A. (1950). Curso de historia del derecho español, tomo I. Madrid: Gráfica Administrativa.

Gibbon, E. (2006). Juicio histórico del derecho romano: capítulo XLIV De la historia de la decadencia y caída del Imperio Romano. Madrid: Marcial Pons.

Goethe, J. W. (1999). Poesía y verdad (De mi vida). España: Alba.

Gómez Rojo, M. E. (2003). Historia del derecho y de las instituciones. Un ensayo conceptual y de fundamentación científica a la luz de la doctrina hispánica y del derecho español y comparado francés, alemán y suizo. Málaga: Universidad de Málaga.

Grossi, P. (2008). Europa y el derecho. Madrid: Crítica (traducción del italiano 2007).

Herodoto (1984). Historia. Madrid: Gredos.

Huizinga, J. (1994). El concepto de la historia. México: FCE (1 a ed. castellano, 1946).

Iglesias Ferreirós, A. (1996). La creación del derecho. Una historia de la formación de un derecho estatal español. Manual I. Madrid: Marcial Pons.

Luciano de Samósata (1990). Cómo debe escribirse la historia. En Obras III (pp.367-385). Traducción y notas de Juan Botella Zargoza. Madrid: Gredos.

Montanos Ferrín, E. y Sánchez-Arcil, J. (1991). Historia del derecho y de las instituciones, tomo I. Madrid: Dykinson.

Polibio (1981). Historias. Madrid: Gredos.

Rodríguez Medina, J. I. (2010, septiembre 5). Entrevista: "Habla el nuevo Premio Nacional de Historia, Bernardino Bravo". Diario El Mercurio (Santiago), Artes y Letras, E5.

Sala, R. (1999). “Introducción”. En Goethe, J. W. Poesía y verdad (De mi vida). España: Alba.

Zorraquín Becú, R. (1992). "Apuntes para una Teoría de la Historia del Derecho". Estudios de Historia del Derecho III. Instituto de Investigaciones de Historia del Derecho. Buenos Aires: Beledo-Perrot. 